\title{
Bioanalysis
}

\section{Microextraction techniques in illicit drug testing: present and future}

\author{
"Sample clean-up is a difficult step that takes $50-75 \%$ of the total \\ analysis time and remains one of the main bottlenecks of modern \\ illicit drug testing."
}

First draft submitted: 15 January 2016; Accepted for publication: 16 February 2016; Published online: 20 April 2016

\section{Keywords: biological matrix $\bullet$ dLLME $\bullet$ DPX $\bullet$ illicit drugs $\bullet$ MEPS $\bullet$ microextraction $\bullet$ SPME}

Sample preparation is of utmost importance in both qualitative and quantitative analysis of illicit drugs because complex matrices, such as biological specimens, are involved in the assay. In forensic toxicology, the analysis is typically performed on biological fluids such as urine, serum and plasma. Matrices such as hair, oral fluid (OF), sweat, vitreous humor or meconium have become alternative options in recent times.

Sample clean-up is a difficult step that takes $50-75 \%$ of the total analysis time and remains one of the main bottlenecks of modern illicit drug testing. Conventionally in forensic toxicology, sample preparation is conducted by liquid-liquid extraction (LLE), protein precipitation and solid-phase extraction (SPE). These techniques may involve large amounts of organic solvent and often require complex time-consuming multistep procedures, which can lead to low accuracy and precision, contamination and loss of analytes. For this reason, to address some of the shortcomings of current clean-up procedures, the development of new sample preparation techniques is attractive. Novel sample-preparation procedures are being increasingly used for illicit drug testing in biological matrices.

Scaling down the conventional clean-up techniques is a recent trend in analytical chemistry and it allows the reduction of sample volume and organic solvent, leading to a cheaper, quicker and environment-friendly analysis. This editorial will therefore examine the most commonly used microextrac- tion techniques and their potential in the determination of illicit drugs.

\section{Miniaturized techniques}

Miniaturization of traditional LLE and SPE has led to the development of a number of new methodologies, which involve small volumes of extracting solvent and a minimum number of steps.

\section{Liquid-liquid microextraction}

The liquid-liquid microextraction techniques (LLME) currently most commonly used for the determination of illicit drugs are hollow fiber liquid-phase microextraction (HF-LPME) and dispersive liquid-liquid microextraction (dLLME).

The latter consists in the quick injection of a disperser solvent containing an appropriate amount of an extraction solvent into the aqueous sample. The mixture is then shaken with the consequent formation of a cloudy solution. After centrifugation, the fine particles of extraction solvent are precipitated and collected. Usually, the water-immiscible extracting solvent has a higher density than water, while the disperser solvent should be miscible both in the extracting solvent and the aqueous sample; acetone, acetonitrile and methanol may be used for this purpose [1].

In HF-LPME, the analytes are extracted from aqueous samples, through a thin layer of solvent immobilized within a porous hollow fiber (supported liquid membrane) and into an acceptor solution, which is located inside

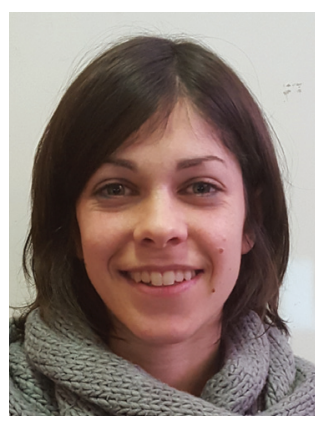

Camilla Montesano Author for correspondence: Department of Chemistry, Sapienza University of Rome, P.le Aldo Moro, 5, 00185 Rome, Italy

Tel.: +39(0)649913559

camilla.montesano@uniroma1.it

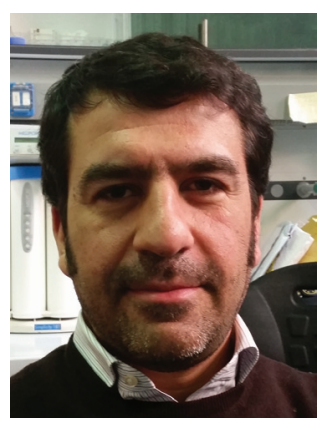

Manuel Sergi

University of Teramo, Faculty of Bioscience \& Technology for Food, Agriculture \& Environment, 64023 Mosciano Sant'Angelo (TE), Italy 
the lumen of the hollow fiber [2]. The microextract is not directly in contact with the sample solution and, therefore, the samples may be stirred without restrictions, to obtain an increased extraction rate, without any loss of the microextract.

\section{Solid-phase microextraction}

Commercial miniaturized SPE devices are available in a variety of formats but solid-phase microextraction (SPME) is certainly the most commonly used [3] and it is today a well-established sample preparation technique. An SPME device consists of a modified syringe containing a fused-silica fiber tip coated with an organic polymer within its needle. Other configurations such as thin film microextraction are possible. Extraction may be performed either by direct immersion of the fiber into a liquid sample (DI-SPME) or by head-space extraction (HS-SPME) in which the fiber is placed just above the sample, which is heated in a vial to volatilize the analytes. Desorption of the analytes may be performed thermally within the GC injector or by a suitable solvent, especially when the extraction is coupled to LC analysis. To allow the extraction of analytes of different polarities there are various fiber coatings commercially available such as polyacrylate, polydimethylsiloxane and polydimethylsiloxane-divinylbenzene. In addition, selective coatings have been developed based on molecularly imprinted polymers or aptamers. The fibers can be reused after a simple wash and reconditioning.

\section{“...miniaturized solid-phase extraction may then be the choice when analyzing oral fluid, blood specimens, vitreous humor and whenever sample volume is limited."}

A further development of SPME is constituted by stir bar sorptive extraction in which the extraction phase is coated onto magnetic stir bars. In comparison to SPME, this may obtain higher recoveries.

\section{Disposable pipette extraction}

Disposable pipette extraction or $\mu$-SPE uses standard pipette tips, which contain a loosely packed sorbent. The sample and the solvents used for washing and elution are taken in or out through the tip, without the need for vacuum, contrary to classic SPE techniques. The same steps involved in traditional SPE are performed in a typical $\mu$-SPE procedure, but the sample is withdrawn/aspirated through the tip several times in order to obtain an effective extraction. A variety of sorbents (e.g., $\mathrm{C}_{18}, \mathrm{C}_{8}$, anion and cation exchange) and tip capacities $(200 \mu \mathrm{l}$ to $5 \mathrm{ml})$ are commercially available.

\section{Microextraction on packed sorbent}

Another novel technique based on SPE microscaling is microextraction on packed sorbent (MEPS) [4]. In MEPS the sorbent bed (of a few milligrams) is inserted into the barrel of a syringe. Similarly to $\mu$-SPE, samples are aspirated and ejected several times to increase extraction efficiency. Otherwise, it is possible to work with large volumes, by aspiring different aliquots from the same vial multiple times in order to obtain a desirable enrichment factor. In MEPS the same sorbent bed can be reused several times without any performance loss by just washing with appropriate solvents. Sorbents typically found in SPE (e.g., C18, C8, SCX and PS-DVB.) can be found with MEPS.

\section{Benefits for illicit drug testing}

Both microsampling SPE and LLE offer the advantage of reducing the volume of solvents used in the process and allow to minimize the manual operations usually associated with sample preparation, reducing the time required for extraction.

When SPE is conducted on a microformat the volume of the sample may be strongly reduced, miniaturized SPE may then be the choice when analyzing OF, blood specimens, vitreous humor and whenever sample volume is limited. Even with urine, which is more readily available, the use of a low volume may be convenient. Taking into consideration that multiclass analysis is not always possible and a number of different analysis may be required on a single sample, limiting the available volume for each assay. In addition when enzymatic hydrolysis is required, low sample volumes mean that there is a reduction in the volume of enzyme required, which consequently leads to reduced analysis costs [5].

On the other hand with LLME, the sample volume is similar to the traditional techniques $(0.5-1 \mathrm{ml})$ but a very low amount of solvent is used, resulting in considerable enrichment factors for the tested compounds without the need for time-consuming evaporation and reconstitution steps. This may be a desirable result when the tested drugs are in low concentrations, such as acid drugs in $\mathrm{OF}$ and cannabinoids in urine (cut-off at $15 \mathrm{ng} / \mathrm{ml}$ ).

Last but not least, the benefits of using microextraction techniques in forensic toxicology also concern the possibility of making life easier to the analysts themselves. For example, when compared with classic SPE, DPX or MEPS techniques allow laboratory throughput improvements; in practicality by using automatic pipettes or syringes several specimens can be processed almost simultaneously without the need to apply vacuum. In addition MEPS provides easy automation by directly inserting the syringe in an autosampler. 
SPME is also an easy to follow technique: when coupled to GC-MS analysis, sampling, extraction, concentration and injection are combined into a single step, thus reducing sample-preparation time. Even derivatization may be performed directly on the fiber. When coupled with LC-MS(/MS) extraction of the analytes from the fiber is performed with a solvent, but the whole extraction procedure lends itself for automation; robotic sample-preparation stations are commercially available in multiwell-plate format (typically 96-well) providing high-throughput down to $1.5 \mathrm{~min}$ per sample. Thin film and fiber SPME configurations are both compatible with viscous and complex samples; whole blood or OF may be processed without any pretreatment.

LLME is less amenable to automation; recent advances have been accomplished for the automation of dLLME [6], however an effective and simple automated procedure is still not available.

\section{Where do we stand with microsampling in illicit drug testing?}

In most laboratories, routine analysis of illicit drug is still carried on with the traditional techniques, especially inexpensive LLE and protein precipitation, while SPE is also frequently used and results in better specificity and cleaner extracts. Beyond the conventional techniques, SPME is the most commonly used, even for routine applications both for single classes of analytes and for general screening purposes. Blood, OF, urine and hair extracts may be equally processed by traditional SPME, however, as previously discussed, the latest and most appealing development of this technique is related to its automation. Interesting multiclass methods for illicit drug determination, by automated thin-film SPME in an automated 96-blade format coupled to LC-MS/MS analysis were reported [7,8]. Another exciting application of SPME is the on-line coupling with ambient MS methods, such as DESI. An SPME-DESI-MS/MS method was described for the quantification of therapeutics and drugs of abuse in urine [9].

Application of other microextraction techniques is still limited to research, but they are gaining attention in the forensic laboratories, especially dLLME which may be easily performed in any laboratory without the need of purchasing special devices. Normally single analytes or uniform chemical classes are extracted, however dLLME multiclass methods have also been reported for blood samples [10]. Even if when compared with dLLME, HF-LPME [11] shows higher precision, more effective sample clean-up, higher extraction efficiency and lower organic solvent consumption, it is probably more intricate and it is therefore less suitable for routine analysis.
MEPS and $\mu$-SPE have been less used, however both the techniques were shown to be suitable for multiclass analytes determination in plasma, saliva and urine by LC-MS/MS [12,13], with a few simple steps obtaining optimal recoveries and great matrix effect reduction. DPX was also described in the 96-well plates format for high-throughput cleanup [14]. An additional improvement of MEPS for OF testing has been described in a recent article and consists of the introduction of a catheter to aspirate saliva directly from the mouth into the MEPS syringe [15].

\section{Future perspective}

Microextraction techniques such as dLLME, SPME, DPX and MEPS are increasingly being used in sample preparation for illicit drug testing but their application has been predominantly limited to research, with infrequent usage for routine analysis.

We believe that because of their simplicity, quickness and cost-effectiveness, microextraction techniques will become a popular choice for forensic analysts especially when the samples have a limited volume and/or when low concentrations must be detected.

The discussed miniaturized techniques will become even more appealing when complete automation will be possible and robotic stations will become commercially available, providing, besides shorter analysis time, better reproducibility and accuracy.

The development of new sorbent materials with increased specificity for miniaturized SPE could also be a future prospect, but it is in contrast with the need for multiclass methods, which require to manage several molecules with different physical-chemical properties.

The rapid diffusion of new psychoactive substances, which are sold in specialized 'head shops' as well as via the internet under the guise of incense, bath salts or standards for laboratory, need the development of new analytical methods; as such, extraction (and especially microextraction) procedures have to take into account the need for flexibility and adaptability in order to include such new analytes.

\section{Financial \& competing interests disclosure}

The authors have no relevant affiliations or financial involvement with any organization or entity with a financial interest in or financial conflict with the subject matter or materials discussed in the manuscript. This includes employment, consultancies, honoraria, stock ownership or options, expert testimony, grants or patents received or pending, or royalties.

No writing assistance was utilized in the production of this manuscript. 


\section{References}

1 Zgoła-Grześkowiak A, Grześkowiak T. Dispersive liquidliquid microextraction. TrAC Trends Anal. Chem. 30(9), 1382-1399 (2011).

2 Rasmussen KE, Pedersen-Bjergaard S. Developments in hollow fibre-based, liquid-phase microextraction. $\operatorname{Tr} A C$ Trends Anal. Chem. 23(1), 1-10 (2004).

3 Arthur CL, Pawliszyn J. Solid-phase microextraction with thermal-desorption using fused-silica optical fibers. Anal. Chem. 62(19), 2145-2148 (1990).

4 Abdel-Rehim M. New trend in sample preparation: on-line microextraction in packed syringe for liquid and gas chromatography applications. I. Determination of local anaesthetics in human plasma samples using gas chromatography-mass spectrometry. J. Chromatogr. B Analyt. Technol. Biomed. Life Sci. 801(2), 317-321 (2004).

5 Montesano C, Sergi M, Odoardi S, Simeoni MC, Compagnone D, Curini R. A mu-SPE procedure for the determination of cannabinoids and their metabolites in urine by LC-MS/MS. J. Pharm. Biomed. Anal. 91, 169-175 (2014).

6 Maya F, Estela JM, Cerda V. Completely automated in-syringe dispersive liquid-liquid microextraction using solvents lighter than water. Anal. Bioanal. Chem. 402(3), 1383-1388 (2012).

7 Boyaci E, Gorynski K, Rodriguez-Lafuente A, Bojko B, Pawliszyn J. Introduction of solid-phase microextraction as a high-throughput sample preparation tool in laboratory analysis of prohibited substances. Anal. Chim. Acta 809, 69-81 (2014).

8 Reyes-Garcés N, Bojko B, Pawliszyn J. High throughput quantification of prohibited substances in plasma using thin film solid phase microextraction. J. Chromatogr. 1374, 40-49 (2014).
9 Kennedy JH, Aurand C, Shirey R, Laughlin BC, Wiseman JM. Coupling desorption electrospray ionization with solidphase microextraction for screening and quantitative analysis of drugs in urine. Anal. Chem. 82(17), 7502-7508 (2010).

10 Odoardi S, Fisichella M, Romolo FS, Strano-Rossi S. Highthroughput screening for new psychoactive substances (NPS) in whole blood by DLLME extraction and UHPLC-MS/MS analysis. J. Chromatogr. B Analyt. Technol. Biomed. Life Sci. 1000, 57-68 (2015).

11 Saraji M, Khalili Boroujeni M, Hajialiakbari Bidgoli AA. Comparison of dispersive liquid-liquid microextraction and hollow fiber liquid-liquid-liquid microextraction for the determination of fentanyl, alfentanil, and sufentanil in water and biological fluids by high-performance liquid chromatography. Anal. Bioanal. Chem. 400 (7), 2149-2158 (2011).

12 Montesano C, Simeoni MC, Curini R, Sergi M, Lo Sterzo $\mathrm{C}$, Compagnone D. Determination of illicit drugs and metabolites in oral fluid by microextraction on packed sorbent coupled with LC-MS/MS. Anal. Bioanal. Chem. 407(13), 3647-3658 (2015).

13 Napoletano S, Montesano C, Compagnone D et al. Determination of illicit drugs in urine and plasma by microSPE followed by HPLC-MS/MS. Chromatographia 75(1), 55-63 (2012).

14 Ahsman MJ, Van Der Nagel BC, Mathot RA. Quantification of midazolam, morphine and metabolites in plasma using 96-well solid-phase extraction and ultra-performance liquid chromatography - tandem mass spectrometry. Biomed. Chromatogr. 24(9), 969-976 (2010).

15 Abdel-Rehim A, Abdel-Rehim M. Screening and determination of drugs in human saliva utilizing microextraction by packed sorbent and liquid chromatography-tandem mass spectrometry. Biomed. Chromatogr. 27(9), 1188-1191 (2013). 